\begin{tabular}{|c|l|}
\hline Title & Classifying singular Legendre curves by contactomorphisms \\
\hline Author(s) & Ishikawa, G. \\
\hline Citation & Hokkaido University Preprint Series in Mathematics, 565, 1-17 \\
\hline Issue Date & 2002-10 \\
\hline DOI & 10.14943/83710 \\
\hline Doc URL & http://hdl.handle.net/2115/69314 \\
\hline Type & bulletin (article) \\
\hline File Information & pre565.pdf \\
\hline
\end{tabular}

Instructions for use 
Classifying singular Legendre curves by contactomorphisms

Go-o Ishikawa

Series \#565. October 2002 


\section{HOKKAIDO UNIVERSITY PREPRINT SERIES IN MATHEMATICS}

\#539 M. Jinzenji, Gauss-Manin system and the virtual structure constants, 25 pages. 2001.

\#540 H. Ishii and T.Mikami, Motion of a graph by $R$-curvature, 28 pages. 2001.

\#541 M. Jinzenji and T. Sasaki, $\mathcal{N}=4$ supersymmetric Yang-Mills theory on orbifold $-T^{4} / \mathbf{Z}_{2}$ : higher rank case, 17 pages. 2001.

\#542 T. Nakazi, The Nevanlinna counting functions for Rudin's orthogonal functions, 7 pages. 2001.

\#543 K. Sugano, On H-separable extensions of QF-3 rings, 7pages. 2001.

\#544 A. Arai, Non-relativistic limit of a Dirac-Maxwell operator in relativistic quantum electrodynamics, 27pages. 2001.

\#545 O. Sawada, On time-local solvability of the Navier-Stokes equations in Besov spaces, 30 pages. 2001.

\#546 C. M. Elliott, Y. Giga, and S. Goto, Dynamic boundary conditions for Hamilton-Jacobi equations, 27 pages. 2001.

\#547 Y. Nakano, Minimizing coherent risk measures of shortfall in discrete-time models with cone constraints, 22 pages. 2002.

\#548 K. tachizawa, A generalization of the Lieb-Thirring inequalities in low dimensions, 13 pages. 2002.

\#549 T. Nakazi, Absolute values and real parts for functions in the Smirnov class, 8 pages. 2002.

\#550 T. Nakazi and T. Watanabe, Properties of a Rubin's orthogonal function which is a linear combination of two inner functions, 9 pages. 2002.

\#551 T. Ohtsuka, A level set method for spiral crystal growth, 24 pages. 2002.

\#552 M.-H. Giga and Y. Giga, Minimal vertical singular diffusion preventing overturning for the Burgers equation, 18 pages. 2002.

\#553 Y. Giga and P. Rybka, Berg's effect, 12 pages. 2002.

\#554 Y. Tonegawa, Domain dependent monotonicity formula for a singular perturbation problem, 14 pages. 2002.

\#555 S. Izumiya and N. Takeuchi, New special curves and developable surfaces, 10 pages. 2002.

\#556 S. Izumiya, K. Saji and N. Takeuchi, Singularities of line congruences, 17 pages. 2002.

\#557 S. Izumiya, D. Pei and M. C. Romero Fuster, The lightcone Gauss map of a spacelike surface in Minkowski 4-space, 21 pages. 2002.

\#558 S. Izumiya, D. Pei and M. C. Romero Fuster, Umbilicity of spacelike submanifolds of Minkowski space, 14 pages. 2002.

\#559 S. Izumiya, D. Pei and M. Takahashi, Curves and surfaces in Hyperbolic space, 16 pages. 2002.

\#560 J. Kato, On the uniqueness of nondecaying solutions for the Navier-Stokes equations, 19 pages. 2002.

\#561 M. Jinzenji and T. Sasaki, An Approach to $\mathcal{N}=4 A D E$ gauge Theory on $K 3,29$ pages. 2002

\#562 T.Nakazi and T.Yamamoto, Norms of some singular integral operators on weighted $L^{2}$ spaces, 27 pages. 2002

\#563 A.Harris and Y.Tonegawa, A $\bar{\partial} \partial$-poincaré lemma for forms near an isolated complex singularity, 8 pages. 2002

\#564 M.Takahashi, Bifurcations of ordinary differential equations of Clairaut type, 23 pages.2002 


\title{
Classifying singular Legendre curves by contactomorphisms
}

\author{
Go-o ISHIKAWA*
}

\section{Introduction.}

It is well known that all Legendre curves in contact three spaces are locally equivalent via contactomorphisms; the relative Darboux theorem [2]. We can try to generalize this fact into two directions; global and singular ways. To the global direction, we see the classification of Legendre knots as one of main subjects in the contact topology [4][7]. Then it is well known globally the diffeomorphism classification and the contactomorphism classification are different. In fact even trivial knots have non-equivalent contactomorphism types. Now, going to the singular direction, we first encounter the classification of singular Legendre curves by contactomorphisms as one of main subjects in the contact singularity theory [1]. Then in the present paper we give several basic results on the subject in the local and complex situation. In particular, we show the following:

Theorem 1.1 Two integral curves $(\mathbf{C}, 0) \rightarrow\left(\mathbf{C}^{3}, 0\right)$ are contactomorphic if and only if they are diffeomorphic.

In [1] integral curves $f:(\mathbf{C}, 0) \rightarrow\left(\mathbf{C}^{2 n+1}, 0\right)$ with ord $f=2$ are classified by contactomorphisms in general dimensional cases into series $A_{2 k, 0}$ and $A_{2 k, r}$. If $n \geq 2, A_{2 k, 0}$ and $A_{2 k, r}, r>0$ are not contactomorphic while they are diffeomorphic. Thus we see the classification of singular Legendre curves

Key words: integral curves, relative Darboux theorem, finitedeterminacy.

2000 Mathematics Subject Classification: Primary 58K40; Secondly 57R45, 53D10.

* Partially supported by Grants-in-Aid for Scientific Research, No. 14340020. 
$(n=1)$ has the special feature, compared with the higher codimensional cases $(n \geq 2)$.

Now we explane notions, with some motivations, appearing in the above theorem.

Let $P T^{*} \mathbf{C}^{2}$ denote the projectivization of the cotangent bundle $T^{*} \mathbf{C}^{2}$ over $\mathrm{C}^{2}$. It is a trivial $\mathrm{C}^{1}$-bundle over $\mathrm{C}^{2}$ with the canonical projection $\pi: P T^{*} \mathbf{C}^{2} \rightarrow \mathbf{C}^{2}$, and it is identified with the space of contact elements on $\mathrm{C}^{2}$; An element $c=[\alpha] \in P T^{*} \mathrm{C}^{2}$ with $\alpha \in T_{\pi(c)}^{*} \mathbf{C}^{2} \backslash\{0\}$ defines uniquely the tangential line $\operatorname{Ker}(\alpha) \subseteq T_{\pi(c)} \mathbf{C}^{2}$.

The space of contact elements $P T^{*} \mathrm{C}^{2}$ has the complex analytic contact structure (distribution) $D \subseteq T\left(P T^{*} \mathbf{C}^{2}\right)$ defined by $D_{c}:=\pi_{*}^{-1}(c)$ for each $c \in P T^{*} \mathbf{C}^{2}$, where $c$ is regarded also as a contact element $c \subseteq T_{\pi(c)} \mathbf{C}^{2}$, and $\pi_{*}: T\left(P T^{*} \mathbf{C}^{2}\right) \rightarrow T \mathbf{C}^{2}$ denotes the differential of $\pi: P T^{*} \mathbf{C}^{2} \rightarrow \mathbf{C}^{2} . \mathrm{A}$ diffeomorphism $\Phi: V \rightarrow W, V$ and $W$ being open subsets of $P T^{*} \mathbf{C}^{2}$, is called a contact diffeomorphism or briefly a contactomorphism if $\Phi$ preserves the contact structure $D ; \Phi_{*}\left(\left.D\right|_{V}\right)=\left.D\right|_{W}$. By Darboux's theorem, the pseudogroup of local contactomorphisms on $P T^{*} \mathrm{C}^{2}$ acts transitively.

A complex analytic mapping $f: U \rightarrow P T^{*} \mathbf{C}^{2}$ from an open subseteq $U \subseteq$ $\mathrm{C}$ is called an integral curve if $f_{*}(\partial / \partial z) \in D_{f(z)}$. Moreover if $f$ is an integral immersion, then it is called a Legendre curve. Thus by a singular Legendre curve we mean a integral curve which is not necessarily an immersion.

Let us fix a coordinate system $x, y$ on $\mathbf{C}^{2}$ and thus the decomposition $\mathbf{C}^{2}=\mathbf{C} \times \mathbf{C}$. Then consider the affine subspace $\mathbf{C}^{3} \subseteq P T^{*} \mathbf{C}^{2}$ consisting of non-vertical contact elemelents on $\mathbf{C}^{2}$ for the projection $(x, y) \mapsto x$ along the $y$-axis, which has the system of coordinates $x, y$ and $p$ with the contact 1-form $\alpha=d y-p d x$ defining $\left.D\right|_{\mathbf{C}^{3}}$. A curve $f: U \rightarrow \mathbf{C}^{3}$ is integral if and only if $f^{*} \alpha=0$. A complex analytic plane curve on $\mathbf{C}^{2}$ lifts to an integral curve, by taking tangent lines to points on the curve, in $P T^{*} \mathrm{C}^{2}$. At a point with the $x$-axis as the tangent line, the integral lifting defines an integral curve-germ $(\mathbf{C}, 0) \rightarrow\left(\mathbf{C}^{3}, 0\right)$.

Two curves $f, f^{\prime}:(\mathbf{C}, 0) \rightarrow\left(\mathbf{C}^{3}, 0\right)$ are called diffeomorphic (resp. contactomorphic) if $f^{\prime}$ is transformed to $f$ by a holomorphic diffeomorphism (resp. a contactomorphism) $\left(\mathbf{C}^{3}, 0\right) \rightarrow\left(\mathbf{C}^{3}, 0\right)$ up to a parametrization. 
In this paper we only consider germs of complex analytic integral curves having locally injective representatives. ( $\mathcal{A}$-finite germs in the sense of [13]).

If two curves are contactomorphic, then of course they are diffeomorphic. Theorem 1.1 states the converse is true when they are integral. Remark that Theorem 1.1 is not valid without the integrality condition. For example a transverse immersed curve to the contact distribution and a non-singular Legendre curve are locally diffeomorphic but not contactomorphic.

Recently, Zhitomirskii [14] generalizes the relative Darboux theorem to singular varieties in a contact space. We shortly explain Zhitomirskii's result in the complex case.

Let $X \subset\left(\mathbf{C}^{2 n+1}, 0\right)$ be a subset and $\alpha$ a 1 -form on $\left(\mathbf{C}^{2 n+1}, 0\right)$. The residual class $[\alpha]_{X}$ of $\alpha$ modulo the exterior differential ideal generated by functions vanishing over $X$, is called the algebraic restriction of $\alpha$ to $X$. Let $X, X^{\prime} \subset\left(\mathbf{C}^{2 n+1}, 0\right)$. Then the algebraic restrictions of the contact structure to $X$ and $X^{\prime}$ respectively are called diffeomorphic if there exists a diffeomorphism $\Phi:\left(\mathbf{C}^{2 n+1}, X\right) \rightarrow\left(\mathbf{C}^{2 n+1}, X^{\prime}\right)$ and a non-vanishing function $\Lambda:\left(\mathbf{C}^{2 n+1}, 0\right) \rightarrow \mathbf{C} \backslash\{0\}$ such that $\left[\Phi^{*} \alpha\right]_{X}=[\Lambda \alpha]_{X}$. If $\left(\mathbf{C}^{2 n+1}, X\right)$ and $\left(\mathbf{C}^{2 n+1}, X^{\prime}\right)$ are contactmorphic, then the algebraic restrictions of the contact structure to $X$ and $X^{\prime}$ are diffeomorphic in the above sense. Then Zhitomorskii shows the following:

Theorem 1.2 (Zhitomorskii [14]) $\left(\mathbf{C}^{2 n+1}, X\right)$ and $\left(\mathbf{C}^{2 n+1}, X^{\prime}\right)$ are contactmorphic if and only if the algebraic restrictions of the contact structure to $X$ and $X^{\prime}$ are diffeomorphic.

Then, related to his result, we interpret our result Theorem 1.1 in term of the algebraic restriction:

Corollary 1.3 (of Theorem 1.1.) Let $f, f^{\prime}(\mathbf{C}, 0) \rightarrow\left(\mathbf{C}^{3}, 0\right)$ be injective integral map-germs. If $f$ and $f^{\prime}$ are diffeomorphic, then the algebraic restrictions of the contact structure on $\left(\mathbf{C}^{3}, 0\right)$ to $f$ and $f^{\prime}$ respectively are diffeomorphic.

The author does not know the direct proof of Corollary 1.3 without using Theorem 1.1. Also it is interesting to consider the real case. 
In $\S 2$ and $\S 3$, we give preliminaries on contactmorphisms and singular Legendre curves (integal curves). In $\S 4$, we introduce the notion of contact codimension and orbital contact codimension for an integral curve, and show that both are not only cotact invariants but also diffeomorphism invariants of integral curves. Moreover, in fact, there are no infinitesimal difference between the contactomorphism classification and the diffeomorphism classification of integral curves, in $\S 5$. In $\S 6$, we show that any injective integral curve is finitely determined among integral curves. In $\S 7$, we complete the proof of Theorem 1.1. Lastly, in $\S 8$, we give several examples of contact simple integral curves among integral curves, related to symplectically simple plane curves [10].

Throughout this paper, for a holomorphic function $h:(\mathbf{C}, 0) \rightarrow \mathbf{C}$, we denote by ord $h$ the order of $h$ at 0 , the degree of the leading term.

The author would like to thank S. Janeczko and W. Domitrz for the information about the reference [14].

\section{Contactomorphisms.}

A holomorphic diffeomorphism $\Phi: V \rightarrow W, V$ and $W$ being open subsets in $\mathbf{C}^{3}$ endowed with the contact form $\alpha=d y-p d x$, is a contactomorphism if and only if there exists a non-vanishing holomorphic function $\Lambda$ on $V$ satisfying $\Phi^{*} \alpha=\Lambda \alpha$ on $V$.

Example 2.1 Let $\phi:\left(\mathbf{C}^{2}, 0\right) \rightarrow\left(\mathbf{C}^{2}, 0\right)$ be a diffeomorphism of form $\phi(x, y)=$ $(X(x, y), Y(x, y))$ with $\frac{\partial X}{\partial x}(0,0) \neq 0$. Then $\phi$ defines a contactomorphism $\widehat{\phi}:\left(\mathbf{C}^{3}, 0\right) \rightarrow\left(\mathbf{C}^{3}, \widehat{\phi}(0)\right)$ by $\widehat{\phi}(x, y, p):=(X, Y, P)$,

$$
P(x, y, p):=\frac{\frac{\partial Y}{\partial x}+p \frac{\partial Y}{\partial y}}{\frac{\partial X}{\partial x}+p \frac{\partial X}{\partial y}}, \widehat{\phi}(0)=\frac{\frac{\partial Y}{\partial x}(0,0)}{\frac{\partial X}{\partial x}(0,0)} .
$$

For example,

$$
X=x, P=p-a(x), Y=y-\int_{0}^{x} a(\zeta) d \zeta,
$$

defines a contactomorphism satisfying $d Y-P d X=d y-p d x$. 
Example 2.2 Consider the diffeomorphism on $\mathbf{C}^{3}$ defined by $(x, y, p) \mapsto$ $(X, Y, P)$,

$$
X=-p, P=x, Y=y-p x .
$$

Then we have $d Y-P d X=d y-p d x$. So this is a contactomorphism, which is called the Legendre transformation.

The infinitesimal contact transformations on $\left(\mathbf{C}^{3}, \alpha\right), \alpha=d y-p d x$ are given by the contact Hamiltonian vector fields over $\mathbf{C}^{3}$.

Let $H \in \mathcal{O}_{3}$. Then the contact Hamiltonian vector filed over $\mathrm{C}^{3}$ with the Hamiltonian function $H$ is defined by

$$
X_{H}:=\left(\frac{\partial H}{\partial x}+p \frac{\partial H}{\partial y}\right) \frac{\partial}{\partial p}-\frac{\partial H}{\partial p} \frac{\partial}{\partial x}+\left(H-p \frac{\partial H}{\partial p}\right) \frac{\partial}{\partial y}
$$

(cf. [2]). The vector filed $X_{H}$ is characterized by the conditions

$$
\left\langle\alpha, X_{H}\right\rangle=H, L_{X_{H}} \alpha=\lambda \alpha
$$

for some $\lambda \in \mathcal{O}_{3}$, where $\left\langle\alpha, X_{H}\right\rangle$ means the natural paring of a 1-form and a vector field.

We set

$$
V H_{3}:=\left\{X_{H} \mid H \in \mathcal{O}_{3}\right\}
$$

Then $V_{3}$ is an $\mathcal{O}_{3}$ module: For $K \in \mathcal{O}_{3}$ and for $X_{H} \in V H_{3}$, we set $K * X_{H}:=X_{K H}$.

Now we consider the invariant filtration

$$
\mathcal{O}_{3}=m_{3}^{0} \supset m_{3} \supset m_{3}^{2} \supset m_{3}^{3} \supset \cdots
$$

under diffeomorphisms therefore under contactomorphisms. Here $m_{3}:=\{h \in$ $\left.\mathcal{O}_{3} \mid h(0)=0\right\}$ denotes the unique maximal ideal of $\mathcal{O}_{3}$. Also we consider another invariant filtration under contactomorphisms

$$
\mathcal{O}_{3}=I_{0} \supset I_{1} \supset I_{2} \supset I_{3} \supset \cdots
$$

where we set

$$
I_{j}:=\left\{h \in \mathcal{O}_{3} \mid \operatorname{weight}(h) \geq i\right\}, j=0,1,2, \ldots,
$$


setting weight $(x)=1$, weight $(p)=1$, weight $(y)=2$. Remark that, for an $h \in \mathcal{O}_{3}$ and for a contactomorphism $\Phi:\left(\mathbf{C}^{3}, 0\right) \rightarrow\left(\mathbf{C}^{3}, 0\right)$, we have weight $\left(\Phi^{*} h\right)=$ weight $(h)$. Two filtrations $\left\{m_{3}^{j}\right\}$ and $\left\{I_{j}\right\}$ are related by $I_{2 j} \subseteq m_{3}^{j} \subseteq I_{j},(j=1,2, \ldots)$.

Then easily we have:

Lemma 2.3 We have $I_{2 j} * V H_{3}=V H_{3} \cap m_{3}^{j} V_{3},(j=1,2, \ldots)$. In particular $m_{3}^{2} * V H_{3} \subseteq I_{2} * V H_{3}=V H_{3} \cap m_{3} V_{3} \subseteq m_{3} * V H_{3}$.

\section{Singular Legendre curves.}

Let $f:(\mathbf{C}, 0) \rightarrow\left(\mathbf{C}^{3}, 0\right)$ be an integral map-germ. Set $f=(x, y, p)=$ $\left(f_{1}, f_{2}, \varphi\right)$. Then we have $d f_{2}-\varphi d f_{1}=0$, namely, $\frac{d f_{2}}{d t}=\varphi \frac{d f_{1}}{d t}$. Then ord $\varphi=$ ord $f_{2}-$ ord $f_{1}$. Moreover we have

$$
f_{2}(t)=\int_{0}^{t} \varphi(\zeta) \frac{d f_{1}}{d t}(\zeta) d \zeta
$$

Example 3.1 Let $f_{1}=t^{2}, \varphi=t^{2 k+1}$. Then $f_{2}=\frac{2}{2 k+3} t^{2 k+3}$.

We study the infinitesimal deformations of $f$ among integral mappings. Let $\widetilde{\alpha}$ be the natural lifting to $T \mathrm{C}^{3}$ of the 1 -form $\alpha=d y-p d x$ on $\mathbf{C}^{3}$. Let $(x, y, p ; \widetilde{x}, \widetilde{y}, \widetilde{p})$ be the induced coordinates of $T \mathrm{C}^{3}$ from the coordinates $(x, y, p)$ of $\mathbf{C}^{3}$. Then

$$
\widetilde{\alpha}:=d \widetilde{y}-\widetilde{p} d x-p d \widetilde{x}=d(\widetilde{y}-p \widetilde{x})-\widetilde{p} d x+\widetilde{x} d p .
$$

Now set

$$
V I_{f}:=\left\{w:(\mathbf{C}, 0) \rightarrow T \mathbf{C}^{3} \mid \pi \circ w=f, w^{*} \widetilde{\alpha}=0\right\} .
$$

Let $w=\left(f_{1}, f_{2}, \varphi ; \xi, \eta, \psi\right):(\mathbf{C}, 0) \rightarrow T \mathbf{C}^{3}$ belong to $V I_{f}$. Then we have $d \eta=\psi d f_{1}+\varphi d \xi$ and $d(\eta-\varphi \xi)=-\xi d \varphi+\psi d f_{1}$.

Proposition $3.2 V I_{f}$ coincides with the space of infinitesimal integral deformations of $f:$ For a holomorphic vector field $w:(\mathbf{C}, 0) \rightarrow T \mathbf{C}^{3}$ along $f, w$ satisfies $w^{*} \widetilde{\alpha}=0$ if and only if there exists a holomorphic integral deformation $F:(\mathbf{C} \times \mathbf{C}, 0) \rightarrow \mathbf{C}^{3}$ of $f$ with $w=\left.\frac{\partial F}{\partial s}\right|_{s=0}$. 
Proof: That $F$ is an integral deformation means, setting $F=F(t, s)=f_{s}(t)$, that we have $f_{0}=f$ and $f_{s}^{*} \alpha=0$. By differentiating the both sides of the equality $f_{s}^{*} \alpha=0$ by $s$, we have $w^{*} \widetilde{\alpha}=0$. Conversely, for each $w=$ $\left(f_{1}, f_{2}, \varphi ; \xi ; \eta, \psi\right)$, if we set $F(t, s)=\left(f_{1}(t)+s \xi(t), f_{2}(t)+E(t, s), \varphi+s \psi\right)$, where

$$
E(t, s):=s \int_{0}^{t} \varphi(\zeta) \xi^{\prime}(\zeta) d \zeta+s \int_{0}^{t} \psi(\zeta) f_{1}^{\prime}(\zeta) d \zeta+s^{2} \int_{0}^{t} \psi(\zeta) \xi^{\prime}(\zeta) d \zeta
$$

then $F$ is an integral deformation satisfying $w=\left.\frac{\partial F}{\partial s}\right|_{s=0}$.

For a $w=\left(f_{1}, f_{2}, \varphi ; \xi, \eta, \psi\right) \in V I_{f}$, we call $i_{w} \alpha:=\eta-\varphi \xi \in \mathcal{O}_{3}$ the generating function of $w$. Set $k=\eta-\varphi \xi$. Then we see ord $(k-k(0)) \geq$ $\min \left\{\operatorname{ord} f_{1}, \operatorname{ord} \varphi\right\}$. Set $s=\min \left\{\operatorname{ord} f_{1}, \operatorname{ord} \varphi\right\}(=\operatorname{ord} f)$, and set

$$
R_{f}:=\mathbf{R}+m_{1}^{s}
$$

where $m_{1}:=\left\{h \in \mathcal{O}_{3} \mid h(0)=0\right\}$. Let $k \in R_{f}$. Then there exist $\xi$ and $\psi$ with $d k=-\xi d \varphi+\psi d f_{1}$. Moreover setting $\eta=k+\varphi \xi$, we have $k=\eta-\varphi \xi$.

Therefore we see the linear mapping $e: V I_{f} \rightarrow R_{f}$ defined by taking the generating function, $e(w):=i_{w} \alpha$, is surjective. We denote the kernel of the linear mapping $e$ by

$$
V I_{f}^{\prime}:=\left\{w \in V I_{f} \mid i_{w} \alpha=0\right\} .
$$

We see $V I_{f}^{\prime}$ is an $\mathcal{O}_{1}$-submodule of the $\mathcal{O}_{1}$-module $V_{f}$ of all holomorphic vector fields $(\mathbf{C}, 0) \rightarrow T \mathbf{C}^{3}$ along $f$.

Define $w f: V H_{3} \rightarrow V I_{f}$ by $w f\left(X_{H}\right):=X_{H} \circ f$. In fact, if we regard $X_{H}$ as the section $X_{H}:\left(\mathbf{C}^{3}, 0\right) \rightarrow T \mathbf{C}^{3}$, we see $X_{H}^{*} \widetilde{\alpha}=\lambda \alpha$ for some $\lambda \in \mathcal{O}_{3}$. Therefore $\left(X_{H} \circ f\right)^{*} \widetilde{\alpha}=\left(f^{*} \lambda\right)\left(f^{*} \alpha\right)=0$.

Then the generating function of $w f\left(X_{H}\right)$ is equal to $H \circ f$. In fact, if we set $w=\left(f_{1}, f_{2}, \varphi ; \xi ; \eta, \psi\right)$, then

$$
\xi=-\frac{\partial H}{\partial p} \circ f, \eta=H \circ f-\varphi \frac{\partial H}{\partial p} \circ f, \psi=\frac{\partial H}{\partial x} \circ f+\varphi \frac{\partial H}{\partial y} \circ f,
$$

and we see

$$
\eta-\varphi \xi=\left(H \circ f-\varphi \frac{\partial H}{\partial p} \circ f\right)-\varphi\left(-\frac{\partial H}{\partial p} \circ f\right)=H \circ f .
$$


We define an $\mathcal{O}_{3}$ module structure on $V I_{f}$ as follows: For $H \in \mathcal{O}_{3}$ and $w \in V I_{f}$, we define the multiplication

$$
H * w:=f^{*} H \cdot w+\left(i_{w} \alpha\right)\left(X_{H}-H \cdot X_{1}\right) \circ f .
$$

If we set $w=\left(f_{1}, f_{2}, \varphi ; \xi, \eta, \psi\right)$, then the right hand side of the definition becomes

$$
(H \circ f)(\xi, \eta, \psi)+(\eta-\varphi \xi)\left(-\frac{\partial H}{\partial p} \circ f,-\varphi \frac{\partial H}{\partial p} \circ f, \frac{\partial H}{\partial x} \circ f+\varphi \frac{\partial H}{\partial y} \circ f\right) .
$$

Then we have as in [9]:

Proposition 3.3 The space $V I_{f}$ of infinitesimal integral deformations of an integral mapping $f$ is an $\mathcal{O}_{3}$-module by the multiplication defined above. The sequence

$$
0 \longrightarrow V I_{f}^{\prime} \longrightarrow V I_{f} \stackrel{e}{\longrightarrow} R_{f} \longrightarrow 0
$$

is $\mathcal{O}_{3}$-exact. Moreover, setting

$$
V H_{3, f}^{0}=\left\{X_{H} \in V H_{3} \mid H \circ f=0\right\},
$$

we have an $\mathcal{O}_{3}$-exact sequence,

$$
0 \longrightarrow V I_{f}^{\prime} / w f\left(V H_{3, f}^{0}\right) \longrightarrow V I_{f} / w f\left(V H_{3}\right) \longrightarrow R_{f} / f^{*} \mathcal{O}_{3} \longrightarrow 0 .
$$

The following is clear.

Lemma $3.4 I_{2} * V I_{f} \subseteq m_{3} * V I_{f} \subseteq V I_{f} \cap m_{1} V_{f}$.

Let $f:(\mathbf{C}, 0) \rightarrow\left(\mathbf{C}^{3}, 0\right)$ be an integral curve. We have defined an $\mathcal{O}_{3^{-}}$ module homomorphism $w f: V H_{3} \rightarrow V I_{f}$ by $w f\left(X_{H}\right):=X_{H} \circ f$.

We denote by $V_{1}$ the set of germs of holomorphic vector fields over $(\mathbf{C}, 0)$ and define an $\mathcal{O}_{3}$-module homomorphism $t f: V_{1} \rightarrow V I_{f}$ by $t f(v):=f_{*}(v)$. Remark that, since $f$ is integral, we have $t f(v) \in V I_{f}$ and moreover the generating function of $t f(v)$ is equal to 0 ; $e(t f(v))=0$.

Let $w=\left(f_{1}, f_{2}, \varphi ; \xi, \eta, \psi\right)$ belong to $V I_{f}^{\prime}$. Then $\eta-\varphi \xi=0$. Moreover we have $-\xi d \varphi+\psi d f_{1}=0$, namely, we have $\xi \varphi^{\prime}=\psi f_{1}^{\prime}$, so $\psi=\xi\left(\varphi^{\prime} / f_{1}^{\prime}\right)$. 
Assume, using the Legendre transformation (Example 2.2) if necessary, $\operatorname{ord} f_{1} \leq \operatorname{ord} \varphi\left(=\operatorname{ord} f_{2}-\operatorname{ord} f_{1}\right)$. Then $2 \operatorname{ord} f_{1} \leq \operatorname{ord} f_{2}$ and $s=\operatorname{ord} f_{1}$. Then we have $\eta=\varphi \xi=\xi\left(f_{2}^{\prime} / f_{1}^{\prime}\right)$ and $\psi=\xi\left(\varphi^{\prime} / f_{1}^{\prime}\right)$. So we have

$$
\left(\begin{array}{c}
\xi \\
\eta \\
\psi
\end{array}\right)=\xi\left(\begin{array}{c}
1 \\
f_{2}^{\prime} / f_{1}^{\prime} \\
\varphi^{\prime} / f_{1}^{\prime}
\end{array}\right)=\frac{\xi}{f_{1}^{\prime}}\left(\begin{array}{c}
f_{1}^{\prime} \\
f_{2}^{\prime} \\
\varphi^{\prime}
\end{array}\right)
$$

if $\operatorname{ord} \xi \geq \operatorname{ord} f_{1}-1$.

Thus we have

Lemma 3.5 Let $f:(\mathbf{C}, 0) \rightarrow\left(\mathbf{C}^{3}, 0\right)$ be an integral map-germ. If $w=$ $\left(f_{1}, f_{2}, \varphi ; \xi, \eta, \psi\right) \in V I_{f}^{\prime}$ satisfies ord $\xi \geq$ ord $f_{1}-1$, then $w \in t f\left(V_{1}\right)$. If $\operatorname{ord} \xi \geq \operatorname{ord} f_{1}$, then $w \in t f\left(m_{1} V_{1}\right)$.

In particular we have

Lemma 3.6 $w f\left(V H_{3}\right) \cap V I_{f}^{\prime} \subseteq t f\left(m_{1} V_{1}\right) \subseteq t f\left(V_{1}\right)$.

Proof: Suppose $X_{H} \circ f \in V I_{f}^{0}$. This means that $H \circ f=0$. In particular $H(0)=0$. Set $H(x, y, p)=a x+b y+c p+\widetilde{a} x^{2}+\cdots$. Then $H \circ f=$ $a f_{1}+b f_{2}+c \varphi+\widetilde{a} f_{1}^{2}+\cdots$. We may assume ord $f_{1}<\operatorname{ord} \varphi$ as well as that ord $\varphi$ is not a multiple of ord $f_{1}$, by using a contactomorphism preserving the contact form $\alpha$ as in Example 2.1 if necessary. Then we have $a=0, \widetilde{a}=0, \ldots$ and $c=0$. Therefore we have $\frac{\partial H}{\partial p}(0)=0$, and $\operatorname{ord} \xi \geq \operatorname{ord} f_{1}=\operatorname{ord} f$. Therefore, by Lemma 3.5, we have $w \in t f\left(m_{1} V_{1}\right)$.

\section{Contact codimension.}

Now we define the contact codimension of $f$ by

$$
\operatorname{ct}-\operatorname{codim}(f):=\operatorname{dim}_{\mathbf{C}} \frac{V I_{f}}{t f\left(V_{1}\right)+w f\left(V H_{3}\right)} .
$$

Similarly we define the orbital contact codimension of $f$ by

$$
\operatorname{codim}(\mathrm{Ct} f):=\operatorname{dim}_{\mathbf{C}} \frac{V I_{f} \cap m_{1} V_{f}}{t f\left(m_{1} V_{1}\right)+w f\left(V H_{3} \cap m_{3} V_{3}\right)} .
$$


Then we will show:

Proposition 4.1 The contact codimension of an integral curve $f:(\mathbf{C}, 0) \rightarrow$ $\left(\mathbf{C}^{3}, 0\right)$ is equal to $\operatorname{dim}_{\mathbf{C}} \mathcal{O}_{1} / f^{*} \mathcal{O}_{3}$. The orbital contact codimension of $f$ is equal to $\operatorname{dim}_{\mathbf{C}} m_{1} / f^{*} m_{3}^{2}$. In particular the contact codimension and the orbital contact codimension are both diffeomorphism invariants.

Proof: By Proposition 3.3, we have the exact sequence:

$$
0 \longrightarrow \frac{V I_{f}^{\prime}}{t f\left(V_{1}\right)} \longrightarrow \frac{V I_{f}}{t f\left(V_{1}\right)} \longrightarrow R_{f} \longrightarrow 0 .
$$

Then, by Lemma 3.6, we have the exact sequence:

$$
0 \longrightarrow \frac{V I_{f}^{\prime}}{t f\left(V_{1}\right)} \longrightarrow \frac{V I_{f}}{t f\left(V_{1}\right)+w f\left(V H_{3}\right)} \longrightarrow \frac{R_{f}}{f^{*} \mathcal{O}_{3}} \longrightarrow 0 .
$$

Now we have, setting $s=\operatorname{ord} f$, $\operatorname{dim}_{\mathbf{C}} V I_{f}^{\prime} / t f\left(V_{1}\right)=s-1$. On the other hand we see $\operatorname{dim}_{\mathbf{C}} \mathcal{O}_{1} / R_{f}=s-1$. Thus we have

$$
\operatorname{ct-codim}(f)=\operatorname{dim}_{\mathbf{C}} \mathcal{O}_{1} / R_{f}+\operatorname{dim}_{\mathbf{C}} R_{f} / f^{*} \mathcal{O}_{3}=\operatorname{dim}_{\mathbf{C}} \mathcal{O}_{1} / f^{*} \mathcal{O}_{3} .
$$

Similarly we have the exact sequence,

$$
0 \longrightarrow \frac{V I_{f}^{\prime} \cap m_{1} V_{f}}{t f\left(m_{1} V_{1}\right)} \longrightarrow \frac{V I_{f} \cap m_{1} V_{f}}{t f\left(m_{1} V_{1}\right)+w f\left(V H_{3} \cap m_{3} V_{3}\right)} \longrightarrow \frac{m_{1}^{s}}{f^{*} m_{3}^{2}} \longrightarrow 0 .
$$

Since $\operatorname{dim}_{\mathbf{C}} \frac{V I_{f}^{\prime} \cap m_{1} V_{f}}{t f\left(m_{1} V_{1}\right)}=s-1=\operatorname{dim}_{\mathbf{C}} m_{1} / m_{1}^{s}$, we have

$$
\operatorname{codim}(\mathrm{Ct} f)=\operatorname{dim}_{\mathbf{C}} m_{1} / m_{1}^{s}+\operatorname{dim}_{\mathbf{C}} \frac{m_{1}^{s}}{f^{*} m_{3}^{2}}=\operatorname{dim}_{\mathbf{C}} m_{1} / f^{*} m_{3}^{2} .
$$

\section{Contact diffect.}

Now we consider the infinitesimal difference, which may be called the contact diffect, between the diffeomorphism classification and the contactomorphism classification for integral curves $(\mathbf{C}, 0) \rightarrow\left(\mathbf{C}^{3}, 0\right)$. 
Lemma 5.1 For an integral curve $f:(\mathbf{C}, 0) \rightarrow\left(\mathbf{C}^{3}, 0\right)$, we have $w f\left(m_{3} V_{3}\right) \cap$ $V I_{f}^{\prime} \subseteq \operatorname{tf}\left(m_{1} V_{1}\right)$.

Proof: We set $f=\left(f_{1}, f_{2}, \varphi\right)$. We may suppose ord $f=\operatorname{ord} f_{1}$. Let $v=$ $A \frac{\partial}{\partial x}+B \frac{\partial}{\partial y}+C \frac{\partial}{\partial p} \in m_{3} V_{3} ; A(0)=0, B(0)=0, C(0)=0$. Suppose $w f(v) \in V I_{f}^{\prime}$. Then, by $A(0)=0$, we see ord $(A \circ f) \geq \operatorname{ord} f$. Thus, by Lemma 3.5, we see $w f(v) \in t f\left(m_{1} V_{1}\right)$.

Now set

$$
T D(f):=t f\left(m_{1} V_{1}\right)+w f\left(m_{3} V_{3}\right) \cap V I_{f}
$$

and set

$$
T(f):=t f\left(m_{1} V_{1}\right)+w f\left(V H_{3} \cap m_{3} V_{3}\right) .
$$

Then $T D(f)$ represents the tangent space to the orbit of $f$ for diffeomorphisms in the space of integral map-germs, and $T(f)$ represents the tangent space to the orbit of $f$ by contactomorphisms. It is clear that $T(f) \subseteq T D(f)$. Actually we see there exists no contact diffect:

Lemma $5.2 T D(f)=T(f)$.

Proof: Recall the $\mathcal{O}_{3}$-homomorphism $e: V I_{f} \rightarrow \mathcal{O}_{3}$ defined by $e(w)=\eta-\varphi \xi$ for $w=\left(f_{1}, f_{2}, \varphi ; \xi, \eta, \psi\right) \in V I_{f}$. We set

$$
e(T D(f))=\left\{B \circ f-\varphi A \circ f \mid v \in m_{3} V_{3}, w f(v) \in V I_{f}\right\},
$$

representing $v=v=A \frac{\partial}{\partial x}+B \frac{\partial}{\partial y}+C \frac{\partial}{\partial p}$. We claim $e(T D(f)) \subseteq m_{1}^{2 s}$, where $s=\operatorname{ord} f$. We may assume ord $f_{1}<\operatorname{ord} \varphi$. By $d(B \circ f-\varphi A \circ f)=$ $-(A \circ f) d \varphi+(C \circ f) d f_{1}$, we have $\frac{\partial B}{\partial x}(0)=0$. If $(1) \operatorname{ord} \varphi<2 \operatorname{ord} f_{1}$, then moreover we see $\frac{\partial B}{\partial p}(0)=0$. Then $e(w) \in m_{1}^{2 s}$. If (2) $2 \operatorname{ord} f_{1} \leq \operatorname{ord} \varphi$, then, from just $\frac{\partial B}{\partial x}(0)=0$, we have $e(w) \in m_{1}^{2 s}$. Therefore anyway $e(w) \in m_{1}^{2 s}$. So we have $e(T D(f)) \subseteq m_{1}^{2 s}$.

Besides we have $e(T(f))=\left\{H \circ f \mid H \in \mathcal{O}_{3}, X_{H}(0)=0\right\}$. The condition $X_{H}(0)=0$ is equivalent to that $\frac{\partial H}{\partial x}(0)=0, \frac{\partial H}{\partial p}(0,0)=0$. Therefore we see $e(T(f))=f^{*} m_{3}^{2}=m_{1}^{2 s}$. 
Thus we have $e(T D(f)) \subseteq e(T(f))$. Since the converse inclusion holds trivially, we have $e(T D(f))=e(T(f))$.

Now let $w \in T D(f)=t f\left(m_{1} V_{1}\right)+w f\left(m_{3} V_{3}\right) \cap V I_{f}$. Take $\widetilde{w} \in T(f)=$ $t f\left(m_{1} V_{1}\right)+w f\left(V H_{3} \cap m_{3} V_{3}\right)$ with $e(\widetilde{w})=e(w)$. This is possible since $e(T D(f))=e(T(f))$. Then $w^{\prime}:=w-\widetilde{w} \in t f\left(m_{1} V_{1}\right)+w f\left(m_{3} V_{3}\right) \cap V I_{f}^{\prime}$. Then, by 5.1 , we see $w^{\prime} \in t f\left(m_{1} V_{1}\right)$. Therefore we have $w$ itself belongs to $T(f)$.

\section{Finite determinacy.}

We observe is the following:

Theorem 6.1 An integral curve $(\mathbf{C}, 0) \rightarrow\left(\mathbf{C}^{3}, 0\right)$ is finitely determined among integral curves for contactomorphisms.

Recall that we assume any integral curves are $\mathcal{A}$-finite. Let $f:(\mathbf{C}, 0) \rightarrow$ $\left(\mathbf{C}^{3}, 0\right)$ be an $\mathcal{A}$-finite map-germ. Then $f$ is $\mathcal{L}$-finite. Then there exists $r \in \mathbf{N}$ such that any map-germ $g:(\mathbf{C}, 0) \rightarrow\left(\mathbf{C}^{3}, 0\right)$ with the $r$-jet $j^{r} g(0)=j^{r} f(0)$ satisfies $g^{*} m_{3} \supseteq m_{1}^{r}([13])$. If we take $r$ sufficiently large if necessary, we have $g^{*} m_{3}^{2} \supseteq m_{1}^{r}$

Assume now moreover $f=\left(f_{1}, f_{2}, \varphi\right)$ and $g=\left(g_{1}, g_{2}, \psi\right)$ are integral and $j^{r} g(0)=j^{r} f(0)$. Set $F_{s}=\left(f_{1}+s\left(g_{1}-f_{1}\right), y, \varphi+s(\psi-\varphi)\right)$, where

$$
y=\int_{0}^{t}\{\varphi(\zeta)+s(\psi(\zeta)-\varphi(\zeta))\} \frac{d\left(f_{1}+s\left(g_{1}-f_{1}\right)\right)}{d t}(\zeta) d \zeta .
$$

Then we see each $F_{s}$ is integral, $j^{r} F_{s}(0)=j^{r} f(0)$ and $F_{0}=f, F_{1}=g$. In particular $F_{s}^{*} m_{3}^{2} \subseteq m_{1}^{r}$. Moreover we set, for each $s_{0} \in \mathbf{C}, F:(\mathbf{C} \times \mathbf{C}, 0) \rightarrow$ $\left(\mathbf{C}^{3} \times \mathbf{C}, 0\right)$ by $F(t, s):=\left(F_{s}(t), s-s_{0}\right)$. Then we have $m_{1}^{r} \mathcal{O}_{2} \subseteq F^{*} m_{3}^{2} \mathcal{O}_{4}$. Now consider the infinitesimal deformation $\frac{d F_{s}}{d s}=\left(g_{1}-f_{1}, \frac{\partial y}{\partial s}, \psi-\varphi\right)$. Since

$$
\begin{aligned}
\frac{\partial y}{\partial s}= & \int_{0}^{t}(\psi(\zeta)-\varphi(\zeta)) \frac{d\left(f_{1}+s\left(g_{1}-f_{1}\right)\right)}{d t}(\zeta) d \zeta \\
& +\int_{0}^{t}\{\varphi(\zeta)+s(\psi(\zeta)-\varphi(\zeta))\} \frac{d\left(g_{1}-f_{1}\right)}{d t}(\zeta) d \zeta
\end{aligned}
$$


belongs to $m_{1}^{r} \mathcal{O}_{2}$, we see $\frac{d F_{s}}{d s}$ belongs to $V I_{F_{s}} \cap m_{1}^{r} V_{F_{s}}$, considering also $s$ as a variable.

Now remark that $V I_{F_{s}} \cap m_{1}^{r} V_{F_{s}} \subseteq t F_{s}\left(m_{1} V_{1}\right)+w F_{s}\left(V H_{3} \cap m_{1} V_{3}\right)$. In fact, let $w \in V I_{F_{s}} \cap m_{1}^{r} V_{F_{s}}$. Then the generating function $e(w)$ belongs to $m_{t}^{r} \mathcal{O}_{t, s} \subseteq F_{s}^{*}\left(m_{x, p, y}^{2} \mathcal{O}_{x, p, y, s}\right)$. There exists an $H \in m_{x, p, y}^{2} \mathcal{O}_{x, p, y, s} \subseteq I_{2} \mathcal{O}_{x, p, y, s}$, $w-X_{H} \circ F \in V I_{F_{s}}^{\prime} \cap w F\left(m_{3} V_{4}\right)$. Thus $w-X_{H} \in t F_{s}\left(m_{t} V_{l, s}\right)$. Therefore we see,

$$
V I_{F} \cap m_{1}^{r} V_{F} \subseteq t F\left(m_{1} V_{2}\right)+w F\left(V H_{3} \cap m_{3} V_{4}\right) .
$$

This implies $f$ is finitely determined among integral curves for contactomorphisms.

\section{Proof of Theorem 1.1.}

The key step we have to overcome is the followings:

Proposition 7.1 Let $f, f^{\prime}:(\mathbf{C}, 0) \rightarrow\left(\mathbf{C}^{3}, 0\right)$ be integral map-germs. If $f$ and $f^{\prime}$ are diffeomorphic, then there exists an integral map-germ $f^{\prime \prime}$ such that $f^{\prime \prime}$ is contactomorphic to $f^{\prime}$ and that $f^{\prime \prime}$ is connected to $f$ through an integral and isotropic family, namely, there exist holomorphic diffeomorphisms $\Phi_{s}$ : $\left(\mathbf{C}^{3}, 0\right) \rightarrow\left(\mathbf{C}^{3}, 0\right)$ and $\sigma_{s}:(\mathbf{C}, 0) \rightarrow(\mathbf{C}, 0)$, depending holomorphically on points $s$ in a connected domain in $\mathbf{C}$ which contains 0 and 1 , such that $f_{s}=\Phi_{s} \circ f \circ \sigma_{s}$ are integral and $f_{0}=f^{\prime \prime}, f_{1}=f$.

Proof: Denote by $C, C^{\prime}$ the images of $f, f^{\prime}$ respectively. Suppose there exists a holomorphic diffeomorphism $\Phi:\left(\mathbf{C}^{3}, 0\right) \rightarrow\left(\mathbf{C}^{3}, 0\right)$ such that $\Phi(C)=C^{\prime}$. Compare $\alpha=d y-p d x$ and $\beta:=\Phi^{*} \alpha$. Then we have $\left.\alpha\right|_{C}=0,\left.\beta\right|_{C}=0$. Remark that also $\beta$ is a contact form on $\left(\mathbf{C}^{3}, 0\right)$. This means that $(\beta \wedge$ $d \beta)(0) \neq 0$.

Now put $\alpha_{s}:=(1-s) \alpha+s \beta$, for $s \in \mathbf{C}$. The condition $\left(\alpha_{s} \wedge d \alpha_{s}\right)(0)=0$ is an algebraic equation, in fact of second order, on $s$. Therefore, outside of a finite set $B \subseteq \mathbf{C}, \alpha_{s}$ is a contact form on $\left(\mathbf{C}^{3}, 0\right)$ satisfying $\left.\alpha_{s}\right|_{C}=0$. Take a path connecting 0 and 1 on $\mathbf{C} \backslash B$. Then there exists a family of diffeomorphisms $\Phi_{s}$ depending holomorphically on a sufficiently thin open neighborhood of the path in $\mathbf{C} \backslash B$. This is proved by the infinitesimal method applied to showing Darboux theorem [2]. Consider the curve $C_{s}:=$ 
$\Phi_{s}(C)$. We have $\Phi_{1}^{*} \alpha=\alpha_{1}=\beta=\Phi^{*} \alpha$. So we have $\left(\Phi_{1} \circ \Phi^{-1}\right)^{*} \alpha=$ $\alpha$. Therefore $\Phi_{1} \circ \Phi^{-1}$ is a contactomorphism. Moreover we have $\left.\alpha\right|_{C_{s}}=$ $\left.\Phi_{s}^{*} \alpha\right|_{C}=\left.\alpha_{s}\right|_{C}=0 . \quad\left(\Phi_{1} \circ \Phi^{-1}\right)\left(C^{\prime}\right)=\Phi_{1}(C)=C_{1}$. We denote by $f^{\prime \prime}$ a parametrization (normalization) of $C_{1}$. Then $f^{\prime \prime}$ is an integral curve and contactomorphic to $f^{\prime}$. Furthermore $f$ and $f^{\prime \prime}$ are connected through an integral and diffeomorphic family.

Consider the integral jet space $J_{I}^{r}(1,3) \subseteq J^{r}(1,3)$ defined by

$$
J_{I}^{r}(1,3):=\left\{j^{r} f(0) \in J^{r}(1,3) \mid f:(\mathbf{C}, 0) \rightarrow\left(\mathbf{C}^{3}, 0\right) \text { is integral }\right\} .
$$

Then $J_{I}^{r}(1,3) \subseteq J^{r}(1,3)$ is a complex analytic submanifold. In fact there is an embedding $J^{r}(1,2) \rightarrow J^{r}(1,3)$ defined by $j^{r}\left(f_{1}, \varphi\right)(0) \mapsto j^{r}\left(f_{1}, f_{2}, \varphi\right)(0)$, where

$$
f_{2}(t):=\int_{0}^{t} \varphi(\zeta) \frac{d f_{1}}{d t}(\zeta) d \zeta
$$

Remark that $j^{r-1}\left(\frac{d f_{2}}{d t}\right)(0)=j^{r-1}\left(\varphi \frac{d f_{1}}{d t}\right)(0)$.

We consider the natural action on $J^{r}(1,3)$ (resp. $J_{I}^{r}(1,3)$ ) of the group $\mathcal{A}^{r}$ (resp. $\mathcal{C T}^{r}$ ) consisting of $r$-jets $\left(j^{r} \sigma(0), j^{r} \Phi(0)\right)$ of parameter transformations $\sigma:(\mathbf{C}, 0) \rightarrow(\mathbf{C}, 0)$ and diffeomorphisms (resp. contactomorphisms) $\Psi:\left(\mathbf{C}^{3}, 0\right) \rightarrow\left(\mathbf{C}^{3}, 0\right)$. Then for any $z \in J_{I}^{r}(1,3)$, the orbit $\mathcal{C} \mathcal{T}^{r} z$ under contactomorphisms is contained in the orbit $\mathcal{A}^{r} z$ under diffeomorphisms as well as in $J_{I}^{r}(1,3)$. Them we show that

Proposition 7.2 For any integral map-germ $f:(\mathbf{C}, 0) \rightarrow\left(\mathbf{C}^{3}, 0\right), \mathcal{A}^{r} j^{r} f(0) \cap$ $J_{I}^{r}(1,3) \subseteq \mathcal{C} \mathcal{T}^{r} j^{r} f(0)$

Proof: Let $z \in \mathcal{A}^{r} j^{r} f(0) \cap J_{I}^{r}(1,3)$. Then $z=j^{r} f^{\prime}(0)$ for some integral mapgerm $f^{\prime}$. Moreover, since $f$ is $\mathcal{A}$-r-determined, $f^{\prime}$ is diffeomorphic to $f$. Then by Proposition 7.1 there exists an integral germ $f^{\prime \prime}$ such that $f^{\prime}$ and $f^{\prime \prime}$ are contactomorphic and $f^{\prime \prime}$ are connected by a diffeomorphic integral family $f_{s}$ to $f$. Sinc $\operatorname{dim} T\left(j^{r} f_{s}(0)\right)$ is independent on $s$ and $\frac{d f_{s}}{d s} \in T D\left(f_{s}\right) \subseteq T\left(f_{s}\right)$ (Lemma 5.2). Thus, by Mather's lemma ([12] pp. 534-535), we see $j^{r} f^{\prime \prime}(0) \in$ $\mathcal{C} \mathcal{T}^{r} j^{r} f(0)$. Therefore also $z=j^{r} f^{\prime}(0)$ belongs to $\mathcal{C} \mathcal{T}^{r} j^{r} f(0)$.

Proof of Theorem 1.1: Let an integral germ $f^{\prime}$ be diffeomorphic to $f$. Then $j^{r} f^{\prime}(0) \in \mathcal{A}^{r} j^{r} f(0) \cap J_{I}^{r}(1,3)$. Hence, by Proposition 7.2, we see $j^{r} f^{\prime}(0) \in$ 
$\mathcal{C} \mathcal{T}^{r} j^{r} f(0)$. Then, there exist an integral germ $f^{\prime \prime \prime}$ such that $j^{r} f^{\prime \prime \prime}(0)=j^{r} f(0)$ and $f^{\prime \prime \prime}$ is contactomorphic to $f^{\prime}$. Since $f$ is $r$-determined by contactomorphisms, we see $f^{\prime \prime \prime}$ and $f$ are contactomorphic. Thus we see $f^{\prime}$ and $f$ are contactomorphic as required.

Example 7.3 Let $f:(\mathbf{C}, 0) \rightarrow\left(\mathbf{C}^{3}, 0\right)$ be an integral curve. Set $f(t)=$ $(x, p, y)=\left(t^{2}, \varphi(t), f_{2}(t)\right)$ and assume ord $\varphi=2 k+1, k=0,1,2, \ldots$ Then $f$ is contactomorphic to $\left(t^{2}, t^{2 k+1}, \frac{2}{2 k+3} t^{2 k+3}\right)$. In fact, both are integral and both are diffoemorphic to say $\left(t^{2}, t^{2 k+1}, 0\right)$, so by Theorem 1.1 we see they are contactomorphic.

\section{Contact simple curves.}

An integral curve $f:(\mathbf{C}, 0) \rightarrow\left(\mathbf{C}^{3}, 0\right)$ is called contact simple among integral curves if only a finitely many contactomorphism classes appear in any integral deformation $F:\left(\mathbf{C} \times \mathbf{C}^{k}, 0\right) \rightarrow\left(\mathbf{C}^{3}, 0\right)$ of $f$. Note that the above notion of simplicity is different from the contact simplicity discussed in [1][14].

We consider the projection $\Pi: \mathbf{C}^{3} \rightarrow \mathbf{C}^{2}$ defined by $(x, p, y) \rightarrow(x, p)$, regarding $\mathbf{C}^{3}$ as a part of the contactification of the symplectic plane $\mathbf{C}^{2}$. If a plane curve $g:(\mathbf{C}, 0) \rightarrow\left(\mathbf{C}^{2}, 0\right)$ is given, then we have an integral curve $\tilde{g}:(\mathbf{C}, 0) \rightarrow\left(\mathbf{C}^{3}, 0\right)$ by

$$
\widetilde{g}(t)=(x, p, y)=\left(g_{1}, g_{2}, \int_{0}^{t} g_{2}(\zeta) \frac{d g_{1}}{d t}(\zeta) d \zeta\right)
$$

Plane curve germs $g, g^{\prime}:(\mathbf{C}, 0) \rightarrow\left(\mathbf{C}^{2}, 0\right)$ are called symplectically equivalent or symplectomorphic if there exist a holomorphic diffeomorphism $\sigma$ : $(\mathbf{C}, 0) \rightarrow(\mathbf{C}, 0)$ and a symplectomorphism $\phi:\left(\mathbf{C}^{2}, 0\right) \rightarrow\left(\mathbf{C}^{2}, 0\right)$ with respect to the complex symplectic (area) form $\omega:=d p \wedge d x$.

Lemma 8.1 If two plane curves $g, g^{\prime}:(\mathbf{C}, 0) \rightarrow\left(\mathbf{C}^{2}, 0\right)$ are symplectomorphic, then $\widetilde{g}$ and $\widetilde{g^{\prime}}$ are contactomorphic.

For the proof, see [1]. Then we have 
Corollary 8.2 If $g:(\mathbf{C}, 0) \rightarrow\left(\mathbf{C}^{2}, 0\right)$ is symplectically simple, then $\widetilde{g}$ is contact simple.

In [10] Corollary 9.8, we have classified symplectically simple plane curves. By its complex counterpart, which is proved in the same way, we have

\section{Corollary 8.3}

$$
\begin{gathered}
A_{2 \ell}: t \mapsto\left(t \mapsto(x, p, y)=\left(t^{2}, t^{2 k+1}, \frac{2}{2 k+3} t^{2 k+3}\right),\right. \\
E_{6}: t \mapsto\left(t \mapsto(x, p, y)=\left(t^{3}, t^{4}, \frac{3}{7} t^{7}\right)\right.
\end{gathered}
$$

and

$$
E_{8}: t \mapsto\left(t \mapsto(x, p, y)=\left(t^{3}, t^{5}, \frac{3}{8} t^{8}\right)\right.
$$

are contact simple.

Remark 8.4 Consider the family of plane curves $g_{\lambda}(t)=\left(t^{3}, t^{7}+\lambda t^{8}\right)$ of type $E_{12}$ having the parameter $\lambda$ as the symplectic moduli. The integral liftings $\widetilde{g_{\lambda}}(t)=\left(t^{3}, t^{7}+\lambda t^{8}, \frac{3}{10} t^{10}+\frac{3}{11} \lambda t^{11}\right)$ are, however, cotactomorphic to each other, privided $\lambda \neq 0$. For also the family of plane curves $g_{\lambda}(t)=\left(t^{4}, t^{5}+\lambda t^{7}\right)$ of type $W_{12}$ having the parameter $\lambda$ as the symplectic moduli, the same phenomena occurs: The list of contactomomorphic classes from $\widetilde{g_{\lambda}}$ consists of $\left(t^{4}, t^{5}+t^{7}, \frac{4}{9} t^{9}+\frac{4}{11} t^{11}\right)$ and $\left(t^{4}, t^{5}, \frac{4}{9} t^{9}\right)$.

\section{References}

[1] V.I. Arnold, First steps of local contact algebra, Canad. J. Math. 51-6 (1999), 1123 1134 .

[2] V.I. Arnold, Givental, Symplectic geometry, in Dynamical systems, IV, 1-138, Encyclopaedia Math. Sci., 4, Springer, Berlin, (2001).

[3] V.I. Arnold, V.V. Goryunov, O.V. Lyashko, V.A. Vasil'ev, Singularity Theory II: Classification and Applications, Encyclopaedia of Math. Sci., Vol. 39, Dynamical System VIII, Springer-Verlag (1993). 
[4] D. Bennequin, Entrelacements et équations de Pfaff, Third Schnepfenried geometry conference, Vol. 1 (Schnepfenried, 1982), Astérisque, 107-108 (1983), 87-161.

[5] J.W. Bruce, T. Gaffney, Simple singularities of mappings $\mathbf{C}, 0 \rightarrow \mathbf{C}^{2}, 0$, J. London Math. Soc., 26 (1982), 465-474.

[6] J. Damon, The unfolding and determinacy theorems for subgroups of $A$ and $K$, Memoirs Amer. Math. Soc., vol.50, No. 306, Amer. Math. Soc. (1984).

[7] Y. Eliashberg, Contact 3-manifolds twenty years since J. Martinet's work, Ann. Inst. Fourier (Grenoble) 42 (1992), no. 1-2, 165-192.

[8] C.G. Gibson, C.A. Hobbs, Simple singularities of space curves, Math. Proc. Camb. Phil. Soc., 113 (1993), 297-310.

[9] G. Ishikawa, Symplectic and Lagrange stabilities of open Whitney umbrellas, Invent. math., 126-2 (1996), 215-234.

[10] G. Ishikawa, S. Janeczko, Symplectic bifurcations of plane curves and isotropic liftings, to appear in Quarterly Journal of Math..

[11] J.N. Mather, Stability of $C^{\infty}$ mappings III: Finitely determined map-germs, Publ. Math. I.H.E.S., 35 (1968), $127-156$.

[12] J.N. Mather, Stability of $C^{\infty}$ mappings IV: Classification of stable germs by $\mathbf{R}$ algebras, Publ. Math. I.H.E.S., 37 (1970), 223-248.

[13] C.T.C. Wall, Finite determinacy of smooth map-germs, Bull. London Math. Soc., 13 (1981), 481-539.

[14] M. Zhitomirskii, Relative Darboux theorem for singular manifolds and singular curves in a contact space, Preprint.

Go-o Ishikawa,

Department of Mathematics, Hokkaido University,

Sapporo 060-0810, Japan.

e-mail : ishikawa@math.sci.hokudai.ac.jp 